\title{
PHY-Layer Fairness in Amplify and Forward Cooperative Diversity Systems
}

\author{
Diomidis S. Michalopoulos, Student Member, IEEE, and George K. Karagiannidis, Senior Member, IEEE
}

\begin{abstract}
We deal with the concept of physical-layer fairness in amplify and forward cooperative diversity systems, which reflects the need for equally allocating the consumed power among the relays. To this end, we propose a method which utilizes knowledge on both the instantaneous and average channel conditions in order to encompass this concept, by attributing a weight coefficient to each relay depending on its average channel state and then selecting the relay with the best instantaneous "weighted" channel conditions. We also provide a performance analysis of the proposed scheme that includes an analytical expression for the outage probability, together with a closed form one in the high signal-to-noise-ratio (SNR) regime. Through the latter expression, the average Symbol Error Probability (ASEP) for high SNRs is also derived. Numerical results demonstrate that, for small number of available relays or for high SNRs, the performance of the proposed scheme resembles that of the "best relay selection" scheme, in terms of outage probability and ASEP, despite maintaining the average power consumptions equal.
\end{abstract}

Index Terms-Cooperative diversity, Fading channels.

\section{INTRODUCTION}

$\mathbf{C}$ OOPERATIVE diversity (a.k.a. user cooperating diversity) has recently become an attractive field for researchers devoted to several areas of research [1]- [13], due to the variety of subjects that encompasses in the different levels of Open System Interconnection (OSI). Based upon information sharing among users, it represents an effective solution for future cellular, wireless LAN or ad-hoc wireless communications systems, promising extended coverage and better Quality of Service (QoS) without the need for high transmitting powers. The main advantage of cooperative diversity stems from the broadcast nature of the wireless medium, along with its ability to achieve diversity through independent channels. The cooperating terminals (which are sometimes called partners [9]- [10]) create spatial diversity for each other, hence they are able to form virtual antenna arrays which exploit all the benefits that multiple transmission/reception entails.

Manuscript received October 13, 2006; revised June 6, 2007 and October 2, 2007; accepted October 06, 2007. The associate editor coordinating the review of this paper and approving it for publication was R. Berry. This work was conducted within the framework of the Reinforcement Program of Human Research Manpower (PENED'03), partially funded by the E.U.European Social Fund (75\%) and the Greek Ministry of Development (25\%). This paper was presented in part in ICC'07.

The authors are with the Wireless Communications Systems Group (WCSG), Electrical and Computer Engineering Department, Aristotle University of Thessaloniki, 54124 Thessaloniki, Greece (e-mail: \{dmixalo, geokarag\}@auth.gr).

Digital Object Identifier 10.1109/TWC.2008.060825.

\section{A. Motivation}

Despite these benefits, however, obligating the existing users of a network to serve as the elements of the virtual antenna array as mentioned above, creates a number of newlyappeared problems regarding the efficiency of this scheme, especially in terms of energy usage. To be more precise, due to the fact that cooperative diversity involves joint transmission of the information signal generated from a single user, in order to enhance the QoS of this specific user, it goes without saying that it also involves usage of the remaining cooperating users' energy, which they involuntarily waste for the sake of a partner. It naturally follows then that user cooperating diversity needs also to be studied and thereby designed from a fairness point of view. Regarding the physical layer (PHY-layer), the concept of fairly dealing with the potential relays is in some way analogous with requiring from them equal amount of energy to consume. To this end, the main scope of this work is to introduce the concept of fairness at the PHY-layer in amplify and forward $(A F)$ cooperative diversity systems, and thereafter to provide a method of equally allocating the total consumed energy among the relays.

\section{B. Related Work and Results}

Several works have been published in the past dealing with cooperative diversity systems. Initially, the concept of relaying in wireless channels was introduced by van der Meulen in [7], and then further developed by Cover and El Gamal in [8]. Later, Sendonaris et. al introduced in [9]- [10] the concept of users cooperation as a new form of spatial diversity, and showed that such cooperation leads to both an increase in capacity and robustness to channel variations. In [1]- [2], lowcomplexity cooperative diversity protocols were developed and analyzed, along with an extensive study of the outage behavior of the most common relaying protocols, namely the AF and the decode and forward (DF), when only a single relay is utilized. An extensive information theoretic analysis regarding the capacity of cooperative diversity systems was conducted in [13], [5], while useful results related to the achievable diversity multiplexing tradeoff in cooperative channels can be found in [3], [11]. Moreover, in [14]- [16] the authors derived useful results regarding the Average Symbol Error Probability (ASEP) of cooperative networks with more than one relaying terminal, when operating over Rayleigh fading channels.

Based upon local measurements of the instantaneous channel conditions, in [12] a distributed method of selecting the best relay from a set of $L$ available ones, was developed. As a result, the authors showed that diversity gain on the order of $L+1$ can still be achieved, without the need for 
using distributed space-time coding algorithms. Similar results were also derived in [17], where the authors showed that selection cooperation outperforms the Distributed Space Time Coding system proposed in [3] in terms of outage probability, albeit simpler. More recently, in [18]- [19] the authors dealt with fairness in DF energy-constrained cooperative systems, and proposed protocols according to which the partners are selected depending on the power they contributed to or by other nodes.

The main contribution of this paper can be summarized as follows.

- We introduce the concept of PHY-layer fairness in AF cooperative diversity systems; that is to say, the concept of equally dividing the total consumed energy among the relaying terminals. Further, we propose a method for implementing the above concept, by attributing a weight coefficient to each relay depending on its average channel state, and then selecting the relay corresponding to the best instantaneous "weighted" channel conditions.

- We provide an outage analysis for the proposed model; in particular, an analytical expression for the outage probability, along with a closed-form one in the high signal-tonoise-ratio (SNR) region are derived, as a function of the average channel conditions and the weight coefficients described above. Based upon this expression, we also derive a closed-form expression for the ASEP, in the high SNR region.

Outline: The remainder of this paper is organized as follows. In Section II, the system under consideration is portrayed, together with the fading assumptions. In Section III, we argue for a relay selection method that attains the PHY-layer fairness, by attributing a weight coefficient to the relays depending on their average channel conditions, whereas in Section IV we describe how these weight coefficients are derived. An outage and ASEP analysis of the proposed method are provided in Section V and VI, respectively. Finally, in Section VII we provide some numerical results regarding the performance of the proposed scheme, while in Section VIII we give our concluding remarks and future extensions.

Notation: Before continuing, some notational comments are in order: The probability of the random event $\mathcal{B}$ is denoted with $\operatorname{Pr}\{\mathcal{B}\}, \Gamma(\cdot)$ denotes the gamma function defined in [20, eq. (8.310.1)], $\tanh ^{-1}(\cdot)$ is the hyperbolic arc tangent of its argument, $Q(\cdot)$ is the Gaussian $Q$-function defined as $Q(x)=(1 / \sqrt{2 \pi}) \int_{x}^{\infty} e^{-t^{2} / 2} d t, \operatorname{Ei}(\cdot)$ denotes the exponential integral Ei and $\Gamma(\cdot, \cdot)$ the incomplete gamma function, defined in [20, eq. (8.211.1)] and [20, eq. (8.350.2)], respectively. $\mathcal{N}(\mu, \sigma)$ represents Gaussian distribution with mean $\mu$ and variance $\sigma, \psi(\cdot)$ denotes the digamma function defined in [20, eq. $(8.360 .1)],{ }_{2} F_{1}(\cdot, \cdot ; \cdot ; \cdot)$ the Gaussian hypergeometric function defined in [20, eq. (9.100)] and $u$ stands for the Euler's constant [20, eq. (8.367)]. We use the notation $f_{X}(\cdot)$, $F_{X}(\cdot)$ and $Y_{X}(\cdot)$ to refer to the probability density function (PDF), cumulative density function (CDF) and complementary $\mathrm{CDF}$ of the random variable $X$, respectively. The modified Bessel function of second kind of order $v$ is denoted with $K_{v}(\cdot)$. Finally, the overbar $\left(^{\cdot}\right)$ represents expectation, $E_{Z}\langle\cdot\rangle$ denotes expectation with respect to $Z$, and $(x)^{+}=\max (0, x)$.

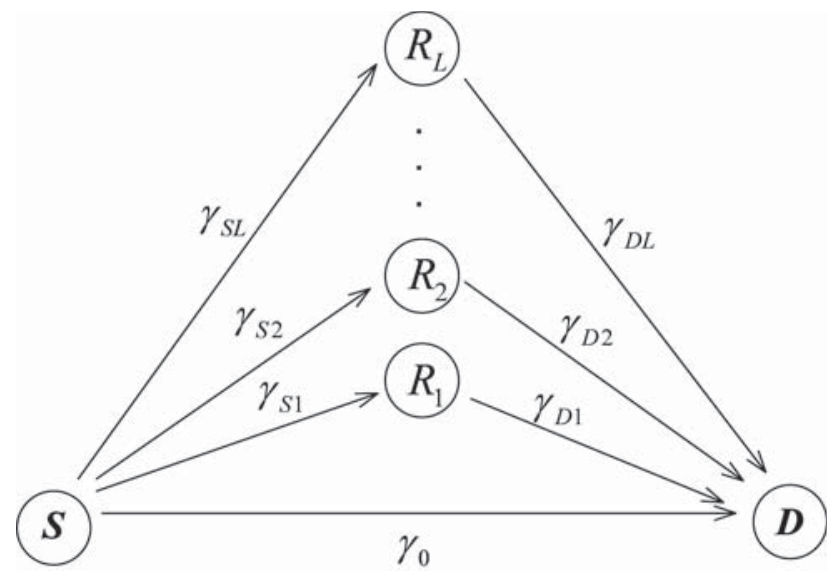

Fig. 1. Typical cooperative diversity system.

\section{SyStem SetuP}

Fig. 1 depicts the typical cooperative diversity setup, where a set of $L$ relays are available for forwarding the information signal received from the source node to the destination one, each one employing a single transmit/receive antenna. The system is considered centralized; in particular, a central unit (CU) exists (which could be e.g., the destination terminal), which knows the average channel conditions of all system links. The source, destination and relay terminals are represented respectively by $S, D$, and $R_{i}$, with $i \in\{1, \ldots, L\}$. All relays are considered to operate in the non-regenerative mode, i.e. they amplify and forward the information signal without any further process. The relaying takes place in a time-orthogonal fashion, which was originally proposed in [2]; according to this protocol, each transmission period is divided in two timeslots, corresponding to the $S-R_{i}$ and $R_{i}-D$ communication interval, respectively. Then, at the end of the second timeslot, the destination terminal combines the signal received directly from the source and that received via the relay (which also contains a packet with $S-R_{i}$ channel state information (CSI)), using a maximal ratio combiner (MRC).

Let us denote with $P_{S}$ the power transmitted by the source ${ }^{1}$. Further, we assume that the gain that each relay employs is that proposed in [1], where the relays actually invert the degradation induced by the corresponding $S-R_{i}$ link in order to maintain their output power limited, i.e.,

$$
G_{i}^{2}(t)=\frac{P_{i}}{a_{S i}^{2}(t) P_{S}+N_{0}},
$$

where $P_{i}$ is the power transmitted by $R_{i}$ and $a_{S i}(t), N_{0}$ denote respectively the instantaneous channel gain of the $S-R_{i}$ link and the additive white Gaussian noise (AWGN) power, which is assumed identical throughout the system. An upper bound of the $S$-D end-to-end SNR associated with the $i$ th relay is [14]- [21]

$$
\gamma_{i}=\frac{\gamma_{S_{i}} \gamma_{D_{i}}}{\gamma_{S_{i}}+\gamma_{D_{i}}}
$$

\footnotetext{
${ }^{1}$ Without loss of generality, we assume constant $P_{S}$, although this model is also applied when non-binary amplitude modulations are used; in such case, $P_{S}$ represents the average transmitting power over the variable bit amplitudes.
} 
where $\gamma_{S_{i}}=P_{S} a_{S i}^{2}(t) / N_{0}$ and $\gamma_{D_{i}}=P_{i} a_{D i}^{2}(t) / N_{0}$ represent the instantaneous SNR corresponding to the $S-R_{i}$ and $R_{i}-D$ channel, respectively. In fact, the end-to-end SNR of (2) corresponds to an ideal relay gain capable of inverting the fading attenuation of the $S-R_{i}$ link ignoring the noise, i.e.,

$$
G_{i}^{2}(t)=\frac{P_{i}}{a_{S i}^{2}(t) P_{S}}
$$

The system under consideration is assumed to operate over independent but not necessarily identically distributed Rayleigh fading channels, that are further assumed to be ergodic and slowly variable. Moreover, all channels are assumed to be quasi-static, that is the fading in each channel remains constant during a coherence time interval and changes independently from one interval to another. Therefore, the instantaneous SNRs at the $S-R_{i}, R_{i}-D$ and $S$-D links are exponentially distributed with probability density function (PDF) given by

$$
f_{Z}(\gamma)=\frac{1}{E\langle Z\rangle} \exp \left(-\frac{\gamma}{E\langle Z\rangle}\right)
$$

where $Z=\left\{\gamma_{S_{i}}, \gamma_{D_{i}}, \gamma_{0}\right\}$ with $\gamma_{0}$ denoting the SNR of the direct $S-D$ channel.

\section{Relay SELECtion Issues}

\section{A. Problem Statement}

In [12], the authors proposed a simple path-selection method which utilizes knowledge on the instantaneous channel gains, in order to activate only the relay which corresponds to the "best" end-to-end SNR between source and destination. Apparently, such technique exploits the ability of achieving diversity through independent wireless channels to the maximum. However, the main drawback of obligating the terminal associated with the best channel conditions act as a relay, is that the additional power consumed by the system as a whole, for the sake of a single user, is not uniformly allocated among the potential relays. Hence, such selection method does not take into account the energy that each of the relays may require in order to participate in the cooperative process. To put it another way, considering the increasing need for storing energy in mobile devices, more equitable (in terms of average power consumption) selection algorithms need to be addressed.

\section{B. Selection Implementation}

Considering the above, the proposed relay selection method encompasses the following two fundamental features:

- utilizing CSI regarding the instantaneous channel gains associated with the $S-R_{i}, R_{i}-D$ links, the selection is repeated in a rate ensuring constant fading conditions during each transmission period. An upper bound of this rate is by definition equal to the inverse of the channel coherence time $T_{c}$.

- assuming that the fading statistics in each $S-R_{i}, R_{i}-D$ channel are known to $\mathrm{CU}$, the selection of the participating relay is implemented according to a rule that leads to equal amount of consumed power, in a longterm perspective.
In order to satisfy these requirements, for each available relaying terminal $R_{i}, i \in\{1, \ldots, L\}$ we define

$$
b_{i} \triangleq \frac{\gamma_{i}}{\mu_{i}}
$$

where $\mu_{i}$ is a positive constant associated with $R_{i}$. In fact, the $b_{i}$ 's represent the selection metrics in the proposed selection method, allowing for the terminal with the highest $b_{i}$ to relay during the current transmission period. In other words, the node $R_{\kappa}$ is selected if

$$
\kappa=\arg \max _{i=1, \ldots, L} b_{i} .
$$

The SNR at the output of the MRC in $D$ is therefore expressed as

$$
\gamma_{\text {end }}=\gamma_{0}+\gamma_{\kappa} .
$$

The selection is assumed to be implemented as follows: at the initialization stage, the $\mu_{i}$ 's are sent to the relays by the $\mathrm{CU}$, in order to be able to form their selection metrics. Then, similarly to the selection method presented in [12], the relays decide in a distributed fashion which one would forward the information for the current interval according to (6), without the need for any instantaneous CSI at the source. Specifically, in cases where a direct communication among the relays is possible, each one of them sets a time-counter which is inversely proportional to the corresponding $b_{i}$. As soon as the shortest counter expires, the remaining relays are informed through a flag packet about the presence of the selected relay, which forwards the information-bearing signal to the destination. If, however, the relays are shadowed with one another, the $\mathrm{CU}$ receives a flag packet from the selected relay and then notifies the rest of them to remain idle.

Depending upon average fading conditions only, the $\mu_{i}$ 's are determined at the initialization stage at the $\mathrm{CU}$ before the communication begins, as we will show later in Section IV-B, and remain constant as long as these conditions do not significantly change, in an average sense. Notice that no continuous channel estimation is needed; depending on the propagation environment, the average fading conditions can be estimated using a long training sequence, and continuously improved during the communication period (see also [22], [23]).

The $\mu_{i}$ 's in (5) play the most important role in attaining the desired PHY-layer fairness, since they represent a weight coefficient attributed to each relay in order to control its average selection time along with the corresponding power consumption. As a result, they directly affect the statistics of the selection metrics, resulting in a PDF and cumulative density function (CDF) having respectively the form of (see [14] for the PDF and CDF of $\gamma_{i}$ )

$$
\begin{aligned}
f_{b_{i}}(b)= & \frac{4 b \mu_{i}}{\rho_{i}} \exp \left(-\frac{b \mu_{i} \sigma_{i}}{\rho_{i}}\right) K_{0}\left(\frac{2 b \mu_{i}}{\sqrt{\rho_{i}}}\right) \\
& +\frac{2 b \mu_{i} \sigma_{i}}{\rho_{i} \sqrt{\rho_{i}}} \exp \left(-\frac{b \mu_{i} \sigma_{i}}{\rho_{i}}\right) K_{1}\left(\frac{2 b \mu_{i}}{\sqrt{\rho_{i}}}\right)
\end{aligned}
$$

and

$$
F_{b_{i}}(b)=1-\frac{2 b \mu_{i}}{\sqrt{\rho_{i}}} \exp \left(-\frac{b \mu_{i} \sigma_{i}}{\rho_{i}}\right) K_{1}\left(\frac{2 b \mu_{i}}{\sqrt{\rho_{i}}}\right),
$$

where $\sigma_{i}=\bar{\gamma}_{S_{i}}+\bar{\gamma}_{D_{i}}$ and $\rho_{i}=\bar{\gamma}_{S_{i}} \bar{\gamma}_{D_{i}}$. 


\section{Special Cases}

1) Best Path Selection (BPS): Observing (5), it is apparent that for the special case of $\mu_{1}=\mu_{2}=\ldots=\mu_{L}$, the proposed method reduces to selecting the relay corresponding to the best end-to-end path, which was the case in [12]. Clearly, such choice for the $\mu_{i}$ 's leads to the maximum attainable performance.

2) Equal Selection Probability (ESP): If the $\mu_{i}$ 's are set equal to the average end-to-end SNR values corresponding to the $i$ th relay $\left(\mu_{i}=\bar{\gamma}_{i}\right)$, then our model actually "forces" the available terminals to relay for an identical fraction of time. In order to evaluate $\bar{\gamma}_{i}$, we use [20, eq. (3.353.5)], [20, eq. (6.455.1)], the primal definition of ${ }_{2} F_{1}(\cdot, \cdot ; \cdot ; \cdot)$ given in [20, eq. (9.100)] and the series representation of the natural logarithm [20, eq. (1.511)] to infer

$$
\begin{aligned}
\bar{\gamma}_{i} & =\frac{1}{\rho_{i}} \int_{0}^{\infty} \int_{0}^{\infty} \frac{x y}{x+y} e^{-x / \bar{\gamma}_{S_{i}}} e^{-y / \bar{\gamma}_{D_{i}}} d x d y \\
& =\frac{\left(1 / \bar{\gamma}_{S_{i}}\right)^{2}-\left(1 / \bar{\gamma}_{D_{i}}\right)^{2}+\left(2 / \rho_{i}\right) \ln \left(\bar{\gamma}_{S_{i}} / \bar{\gamma}_{D_{i}}\right)}{\left(1 / \bar{\gamma}_{S_{i}}-1 / \bar{\gamma}_{D_{i}}\right)^{3}} .
\end{aligned}
$$

Note that the ESP scheme does not necessarily lead to equal amount of consumed power, unless identically distributed fading channels are assumed ${ }^{2}$; in the latter case, the BPS system also coincide with the proposed one.

\section{Towards the Equal Energy Consumption Case}

\section{A. Average Relay Power Consumption}

We assume that the power consumed by the relays consists of two parts: the power consumed by the tx/rx radio circuitry, including the power needed for signal reception, and the power consumed for amplification. Since the former part is fixed for each relay, our study focuses on the latter one, which naturally comprises the largest part of the total consumption. In the time-orthogonal AF scenario described in Section II, the relay passes the received signal through a passive, analog delay line, and amplifies/retransmits it at the second timeslot. Therefore, the instantaneous power that the $i$ th potential relay consumes can be defined as

- the difference between the instantaneous transmitted and received power when this difference is positive (or equivalently, when $G_{i}>1$ ). This stems from the fact that the energy conveyed by the received signal is transformed to a voltage transient within the RF circuitry, hence the energy that the relay consumes equals to that needed in order the transient magnitude to reach the desired level.

- zero, otherwise. This stems from the fact that, when $G_{i} \leq 1$, the resultant signal attenuation can be achieved by utilizing a passive electronic circuit (e.g., a voltage divider).

Let us denote with $P_{i n_{i}}, P_{\text {cons }_{i}}$, the instantaneous received and consumed power of the $i$ th relay, respectively. Then, the above statements can be summarized as

$$
P_{\text {cons }_{i}}=\left(P_{i}-P_{i n_{i}}\right)^{+}=P_{i}\left(1-\frac{1}{G_{i}^{2}}\right)^{+} .
$$

\footnotetext{
${ }^{2}$ Although irrelevant with PHY-layer fairness, selecting the relays with equal probability is important in cross-layer fairness studies, where the concept of fairness is also related to the relays' activation time.
}

For medium and high SNRs, from (3) and (11) we infer

$$
P_{\text {cons }_{i}} \approx P_{i}\left(1-a_{S i}^{2}\right)^{+}=P_{i}\left(1-\frac{\gamma_{S_{i}}}{S N R}\right)^{+},
$$

where $S N R$ stands for the common SNR without fading associate with the transmitted power of the source, i.e., $S N R=P_{S} / N_{0}$. Denoting with $\varpi_{i}$ the probability of selecting the $i$ th relay, and with $\operatorname{Pr}\left\{i=\kappa \mid \gamma_{S_{i}}\right\}$ the corresponding conditional selection probability given that $\gamma_{S_{i}}$ is known, the average power consumption of the $i$ th terminal is obtained as

$$
\left.\bar{P}_{\text {cons }_{i}}=E\left\langle P_{\text {cons }_{i}}\right\rangle=\varpi_{i} E\left\langle P_{\text {cons }_{i}} \mid b_{i}\right\rangle \max _{\substack{j \in\{1, \ldots, L\} \\ j \neq i}} b_{j}\right\rangle
$$

$$
\approx P_{i} \int_{0}^{S N R}\left(1-\frac{\gamma_{S_{i}}}{S N R}\right) f_{\gamma_{S_{i}}}\left(\gamma_{S_{i}}\right) \operatorname{Pr}\left\{i=\kappa \mid \gamma_{S_{i}}\right\} d \gamma_{S_{i}} .
$$

An Approximate Expression for the $\bar{P}_{\text {cons }}$ 's in terms of $\mu_{1}, \ldots, \mu_{L}$ : Since the relay selection is implemented according to (6), the conditional selection probability can be expressed using (2) as

$$
\begin{aligned}
& \operatorname{Pr}\left\{i=\kappa \mid \gamma_{S_{i}}\right\}=\int_{0}^{\infty} f_{\gamma_{D_{i}}}(y) \\
& \times \operatorname{Pr}\left\{\left(b_{i}>\max _{\substack{j \in\{1, \ldots, L\} \\
j \neq i}} b_{j} \mid \gamma_{S_{i}}\right) \mid \gamma_{D_{i}}=y\right\} d y \\
& =\int_{0}^{\infty} f_{\gamma_{D_{i}}}(y) \prod_{\substack{j=1 \\
j \neq i}}^{L} F_{b_{j}}\left(\frac{\gamma_{S_{i}} y}{\mu_{i}\left(\gamma_{S_{i}}+y\right)}\right) d y .
\end{aligned}
$$

However, considering the complexity of the integral in (14), a closed-form evaluation of $\operatorname{Pr}\left\{i=\kappa \mid \gamma_{S_{i}}=x\right\}$ with respect to $\mu_{1}, \ldots, \mu_{L}$ seems to be difficult, if not impossible, especially for large $L$. Alternatively, observing that $b_{i}$ represents the half of the harmonic mean $\mathcal{H}_{\gamma_{S_{i}} / \mu_{i}, \gamma_{D_{i}} / \mu_{i}}$ of $\gamma_{S_{i}} / \mu_{i}, \gamma_{D_{i}} / \mu_{i}$ since

$$
b_{i}=\frac{\gamma_{i}}{\mu_{i}}=\frac{\left(\gamma_{S_{i}} / \mu_{i}\right)\left(\gamma_{D_{i}} / \mu_{i}\right)}{\left(\gamma_{S_{i}} / \mu_{i}\right)+\left(\gamma_{D_{i}} / \mu_{i}\right)}=\frac{1}{\left(\gamma_{S_{i}} / \mu_{i}\right)^{-1}+\left(\gamma_{D_{i}} / \mu_{i}\right)^{-1}},
$$

and using the fact that $b_{i}<\min \left(\gamma_{S_{i}} / \mu_{i}, \gamma_{D_{i}} / \mu_{i}\right)$, we can approximate $\operatorname{Pr}\left\{i=\kappa \mid \gamma_{S_{i}}\right\}$ as

$$
\begin{aligned}
& \operatorname{Pr}\left\{i=\kappa \mid \gamma_{S_{i}}\right\}=\operatorname{Pr}\left\{b_{i}>\max _{\substack{j \in\{1, \ldots, L\} \\
j \neq i}} b_{j} \mid \gamma_{S_{i}}\right\} \\
& \approx \operatorname{Pr}\left\{\min \left(\frac{\gamma_{S_{i}}}{\mu_{i}}, \frac{\gamma_{D_{i}}}{\mu_{i}}\right)>\max _{\substack{j \in\{1, \ldots, L\} \\
j \neq i}} \min \left(\frac{\gamma_{S_{j}}}{\mu_{j}}, \frac{\gamma_{D_{j}}}{\mu_{j}}\right) \mid \gamma_{S_{i}}\right\}
\end{aligned}
$$

In other words, instead of evaluating the probability of selecting the relay with the maximum $b_{i}=\mathcal{H}_{\gamma_{S_{i}} / \mu_{i}, \gamma_{D_{i}} / \mu_{i}}$, we evaluate the probability of selecting the relay with the highest upper bound of $b_{i}$. We note that this bound is as tighter as greater the difference between $\bar{\gamma}_{S_{i}}$ and $\bar{\gamma}_{D_{i}}$, a fact which is derived from the harmonic mean's identity to mitigate the effects of the larger terms and tend strongly towards the least ones. Consequently, from (16) we obtain $\operatorname{Pr}\left\{i=\kappa \mid \gamma_{S_{i}}\right\}$ as shown in (17) at the top of next page. 


$$
\begin{aligned}
& \operatorname{Pr}\left\{i=\kappa \mid \gamma_{S_{i}}\right\} \approx \operatorname{Pr}\left\{\min \left(\frac{\gamma_{S_{i}}}{\mu_{i}}, \frac{\gamma_{D_{i}}}{\mu_{i}}\right)>\max _{\substack{j \in\{1, \ldots, L\} \\
j \neq i}} \min \left(\frac{\gamma_{S_{j}}}{\mu_{j}}, \frac{\gamma_{D_{j}}}{\mu_{j}}\right) \mid \gamma_{S_{i}}\right\} \\
& =\int_{0}^{\gamma_{S_{i}}} f_{\gamma_{D_{i}}}(y) \prod_{\substack{j=1 \\
j \neq i}}^{L}\left[1-Y_{\gamma_{S_{j}}}\left(\frac{\mu_{j}}{\mu_{i}} y\right) Y_{\gamma_{D_{j}}}\left(\frac{\mu_{j}}{\mu_{i}} y\right)\right] d y+\int_{\gamma_{S_{i}}}^{\infty} f_{\gamma_{D_{i}}}(y) \prod_{\substack{j=1 \\
j \neq i}}^{L}\left[1-Y_{\gamma_{S_{j}}}\left(\frac{\mu_{j}}{\mu_{i}} \gamma_{S_{i}}\right) Y_{\gamma_{D_{j}}}\left(\frac{\mu_{j}}{\mu_{i}} \gamma_{S_{i}}\right)\right] d y \\
& =\frac{1}{\bar{\gamma}_{D_{i}}} \int_{0}^{\gamma_{S_{i}}} \exp \left(-\frac{y}{\bar{\gamma}_{D_{i}}}\right) \prod_{\substack{j=1 \\
j \neq i}}^{L}\left[1-\exp \left(-\frac{\mu_{j} \sigma_{j}}{\mu_{i} \rho_{j}} y\right)\right] d y+\exp \left(-\frac{\gamma_{S_{i}}}{\bar{\gamma}_{D_{i}}}\right) \prod_{\substack{j=1 \\
j \neq i}}^{L}\left[1-\exp \left(-\frac{\mu_{j} \sigma_{j}}{\mu_{i} \rho_{j}} \gamma_{S_{i}}\right)\right]
\end{aligned}
$$

$$
\begin{aligned}
& \bar{P}_{\text {cons }_{i}} \approx P_{i}\left[1+\bar{\gamma}_{S_{i}} N_{0}\left(\exp \left(-\frac{1}{\bar{\gamma}_{S_{i}} N_{0}}\right)-1\right)+\sum_{k=1}^{L-1}(-1)^{k} \sum_{n=1}^{\left(\begin{array}{c}
L-1 \\
k
\end{array}\right)} \frac{1+\bar{\gamma}_{S_{i}} N_{0}\left(\exp \left(-1 / \bar{\gamma}_{S_{i}} N_{0}\right)-1\right)}{1+\bar{\gamma}_{D_{i}} \sum_{l=1}^{k} \varphi_{l, k, n}^{i}}\right. \\
& \left.+\sum_{k=1}^{L-1}(-1)^{k} \sum_{n=1}^{\left(\begin{array}{c}
L-1 \\
k
\end{array}\right)} \frac{\left[\sigma_{i}+\rho_{i} \sum_{l=1}^{k} \varphi_{l, k, n}^{i}+\rho_{i} N_{0}\left(\exp \left(-\frac{\sigma_{i} / \rho_{i}+\sum_{l=1}^{k} \varphi_{l, k, n}^{i}}{N_{0}}\right)-1\right)\right] \sum_{l=1}^{k} \varphi_{l, k, n}^{i}}{\bar{\gamma}_{S_{i}}\left(\bar{\gamma}_{S_{i}}+\rho_{i} \sum_{l=1}^{k} \varphi_{l, k, n}^{i}\right)\left(\sigma_{i} / \rho_{i}+\sum_{l=1}^{k} \varphi_{l, k, n}^{i}\right)^{2}}\right]
\end{aligned}
$$

For simplicity of exposition, let us define

$$
\phi_{j, i} \triangleq \frac{\mu_{j} \sigma_{j}}{\mu_{i} \rho_{j}}
$$

and let $\mathcal{S}_{i}$ represent the set

$$
\mathcal{S}_{i}=\left\{\phi_{j, i}: j \in\{1, \ldots, L\}, j \neq i\right\} .
$$

Furthermore, denote by $\mathcal{S}_{k, n}^{i}$, $\left(k \in\{1, \ldots, L\}, n \in\left\{1, \ldots,\left(\begin{array}{l}L \\ k\end{array}\right)\right\}\right)$ the $n$th $k$-subset of $\mathcal{S}_{i}$, i.e. the $n$th subset of $\mathcal{S}_{i}$ containing exactly $k$ elements; these elements of $\mathcal{S}_{k, n}^{i}$ are denoted with $\varphi_{l, k, n}^{i}(l \in\{1, \ldots, k\})$. Then, the second term within the integral of the right-hand side of (17) can be expressed as

$$
\begin{aligned}
\prod_{\substack{j=1 \\
j \neq i}}^{L}\left[1-e^{-\phi_{j, i} y}\right] & =1+\sum_{k=1}^{L-1}(-1)^{k} \sum_{n=1}^{\left(\begin{array}{c}
L-1 \\
k
\end{array}\right)} \prod_{l=1}^{k} e^{-\varphi_{l, k, n}^{i} y} \\
& =1+\sum_{k=1}^{L-1}(-1)^{k} \sum_{n=1}^{\left(\begin{array}{c}
L-1 \\
k
\end{array}\right)} e^{-y \sum_{l=1}^{k} \varphi_{l, k, n}^{i},}
\end{aligned}
$$

and likewise, substituting $y$ with $\gamma_{S_{i}}$ in (21) yields an alternative expression for the second term of (17). Consequently, from (17) we deduce

$$
\begin{aligned}
\operatorname{Pr}\left\{i=\kappa \mid \gamma_{S_{i}}\right\} & \approx 1+\sum_{k=1}^{L-1} \sum_{n=1}^{\left(\begin{array}{c}
L-1 \\
k
\end{array}\right)} \frac{1-e^{-\gamma_{S_{i}}\left(\frac{1}{\bar{\gamma}_{D_{i}}}+\sum_{l=1}^{k} \varphi_{l, k, n}^{i}\right)}}{(-1)^{k}\left(1+\bar{\gamma}_{D_{i}} \sum_{l=1}^{k} \varphi_{l, k, n}^{i}\right)} \\
& +\sum_{k=1}^{L-1}(-1)^{k} \sum_{n=1}^{\left(\begin{array}{c}
L-1 \\
k
\end{array}\right)} e^{-\gamma_{S_{i}}\left(1 / \bar{\gamma}_{D_{i}}+\sum_{l=1}^{k} \varphi_{l, k, n}^{i}\right)} .
\end{aligned}
$$

Inserting (22) in (13), and after some manipulations we obtain an approximate expression for $\bar{P}_{\text {cons }_{i}}$ given in (18) shown at the top of the page.
Hence, combining (18) and (19) an approximate representation of the $\bar{P}_{\text {cons }_{i}}$ as a function of the $\mu_{i}$ 's is derived. For $L=3$, for instance, $P_{1}$ is obtained directly from (18) by setting $\varphi_{1,1,1}^{1}=\varphi_{1,2,1}^{1}=\mu_{2} \sigma_{2} /\left(\mu_{1} \rho_{2}\right)$ and $\varphi_{1,1,2}^{1}=$ $\varphi_{2,2,1}^{1}=\mu_{3} \sigma_{3} /\left(\mu_{1} \rho_{3}\right)$. The benefit of expressing the $\bar{P}_{\text {cons }_{i}}$ 's as a function of the $\mu_{i}$ 's and elementary functions is that the $\bar{P}_{\text {cons }_{i}}$ 's are more easily processed, as it is needed in order to obtain the desired $\mu_{i}$ 's as it is shown next in Section IV-B. The impact of using the approximate expression for the $\bar{P}_{\text {cons }_{i}}$ 's and not the exact one will be discussed later in Section VII.

\section{B. Derivation of the desired $\mu_{i}$ 's}

Having found an expression for the $\bar{P}_{\text {cons }_{i}}$ 's as a function of the $\mu_{i}$ 's and the average fading conditions only, the $\mu_{i}$ 's that lead to equal amount of consumed power are derived by solving the $(L-1) \times(L-1)$ system of simultaneous equations $^{3}$

$$
\bar{P}_{\text {cons }_{1}}=\bar{P}_{\text {cons }_{2}}=\ldots=\bar{P}_{\text {cons }_{L}},
$$

and then sent to the relays in order to form their selection metrics.

We emphasize that such equation-solving procedure does not militate against the implementation of the proposed method in practical applications. This is because the expressions for the $\bar{P}_{\text {cons }_{i}}$ 's provided in eq. (18) involve elementary functions only, hence the $\mu_{i}$ 's can be derived in a relatively short amount of time by using one of the wellknown numerical equation-solving methods, e.g., the NewtonRaphson one. Recall that the $\mu_{i}$ 's depend only on the average channel conditions, hence the equation-solving procedure is performed once at the initialization stage and repeated only

\footnotetext{
${ }^{3}$ Apparently, it is the relation among the $\mu_{i}$ 's that determines the average consumptions $P_{i}$; that is, one of the $\mu_{i}$ 's can take any fixed value (e.g., $\left.\mu_{L}=1\right)$
} 
if the channels' statistics change; for the slow-fading scenario assumed throughout this paper, the latter fact is expected to rarely occur.

\section{Outage Analysis}

Let $P_{\text {out }}\left(\gamma_{t h}\right)$ denote the outage probability associated with the SNR threshold $\gamma_{t h}$ (i.e., the probability that the received SNR falls below $\gamma_{t h}$ ). Given that a single relay is selected, this SNR threshold can be expressed in terms of the target spectral efficiency $r$ through $\gamma_{t h}=2^{2 r}-1$ [1]. It holds

$$
\begin{aligned}
P_{\text {out }}\left(\gamma_{\text {th }}\right) & =\sum_{i=1}^{L} \operatorname{Pr}\left\{b_{i} \geq \max _{\substack{j \in\{1, \ldots, L\} \\
j \neq i}} b_{j} \cap \gamma_{0}+\gamma_{i} \leq \gamma_{\text {th }}\right\} \\
& =\sum_{i=1}^{L}\left[\int_{0}^{\gamma_{t h}} f_{g_{i}}(x) \prod_{\substack{j=1 \\
j \neq i}}^{L} F_{b_{j}}\left(\frac{x}{\mu_{i}}\right) d x\right],
\end{aligned}
$$

where $g_{i}=\gamma_{0}+\gamma_{i}$ (with $g_{\kappa}=\gamma_{\text {end }}$ ) and $f_{g_{i}}(\cdot)$ is the PDF of $g_{i}$, which is actually the convolution of $f_{\gamma_{i}}(\cdot)$ and $f_{\gamma_{0}}(\cdot)$, i.e.,

$$
\begin{aligned}
& f_{g_{i}}(x)=\int_{0}^{x} f_{\gamma_{i}}(z) f_{\gamma_{0}}(x-z) d z=\frac{1}{\bar{\gamma}_{0}} \int_{0}^{x}\left[\frac{4 z}{\rho_{i}} e^{-\frac{z \sigma_{i}}{\rho_{i}}}\right. \\
& \left.\times K_{0}\left(\frac{2 z}{\sqrt{\rho_{i}}}\right)+\frac{2 z \sigma_{i}}{\rho_{i} \sqrt{\rho_{i}}} e^{-\frac{z \sigma_{i}}{\rho_{i}}} K_{1}\left(\frac{2 z}{\sqrt{\rho_{i}}}\right)\right] e^{\frac{z-x}{\bar{\gamma}_{0}}} d z .(25)
\end{aligned}
$$

Therefore, the outage probability is derived by substituting (25) and (9) into (24).

\section{A. High SNR Approximation}

Using the approximations $K_{0}(z) \approx-\ln (z)$ and $K_{1}(z) \approx$ $1 / z$, for $z<<1$ [24, eq. (9.6.8) and (9.6.9)], $f_{g_{i}}(x)$ can be written for relatively small values of $x\left(x<<\sqrt{\rho_{i}}, i \in\right.$ $\{1, \ldots, L\})$ as

$$
\begin{aligned}
f_{g_{i}}(x) & =\frac{1}{\bar{\gamma}_{0}} \int_{0}^{x}\left[\frac{\sigma_{i}}{\rho_{i}} e^{-\frac{z \sigma_{i}}{\rho_{i}}}-\frac{4 z}{\rho_{i}} e^{-\frac{z \sigma_{i}}{\rho_{i}}} \ln \left(\frac{2 z}{\sqrt{\rho_{i}}}\right)\right] e^{\frac{1}{\bar{\gamma}_{0}}} d z \\
& =\frac{e^{-\frac{x}{\bar{\gamma}_{0}}}}{\bar{\gamma}_{0}}\left[\frac{1-e^{-\left(\frac{\sigma_{i}}{\rho_{i}}-\frac{1}{\bar{\gamma}_{0}}\right) x}}{1-\frac{\rho_{i}}{\bar{\gamma}_{0} \sigma_{i}}}-\frac{4 I_{1}\left(\frac{2}{\sqrt{\rho_{i}}}, \frac{\sigma_{i}}{\rho_{i}}-\frac{1}{\bar{\gamma}_{0}}, x\right)}{\rho_{i}}\right]
\end{aligned}
$$

where we have introduced the auxiliary function $I_{1}(\cdot, \cdot, \cdot)$ as (please refer to Appendix)

$$
\begin{aligned}
& I_{1}(\delta, b, \omega)=\int_{0}^{\omega} x \ln (\delta x) e^{-b x} d x=-\frac{\ln \frac{b}{\delta}+u-1}{b^{2}} \\
& -\frac{e^{-b \omega}[1+(1+b \omega) \ln (\delta)]+\Gamma(0, b \omega)+\ln (\omega) \Gamma(2, b \omega)}{b^{2}}
\end{aligned}
$$

Substituting (26) and (9) in (24), and using again the approximations in [24, eq. (9.6.8) and (9.6.9)] we obtain

$$
\begin{aligned}
& P_{\text {out }}\left(\gamma_{\text {th }}\right)=\sum_{i=1}^{L} \int_{0}^{\gamma_{\text {th }}} \frac{e^{-\frac{x}{\bar{\gamma}_{0}}}}{\bar{\gamma}_{0} \rho_{i}} \\
& \times\left[\frac{1-e^{-\eta_{i} x}}{\eta_{i} / \sigma_{i}}-4 I_{1}\left(\frac{2}{\sqrt{\rho_{i}}}, \eta_{i}, x\right)\right] \prod_{\substack{j=1 \\
j \neq i}}^{L}\left[1-e^{-\frac{x \mu_{j} \sigma_{j}}{\mu_{i} \rho_{j}}}\right] d x
\end{aligned}
$$

$$
\begin{aligned}
P_{e} & =B \operatorname{Pr}\left\{X>\sqrt{\beta \gamma_{\text {end }}}\right\}=B \operatorname{Pr}\left\{\gamma_{\text {end }}<\frac{X^{2}}{\beta}\right\} \\
& =B \int_{0}^{\infty} P_{\text {out }}\left(\frac{X^{2}}{\beta}\right) f_{X}(X) d X
\end{aligned}
$$


$\Xi_{i}\left(\gamma_{t h}\right)=\frac{\sigma_{i}}{\rho_{i} \eta_{i}}\left[\bar{\gamma}_{0}\left(1-e^{-\frac{\gamma_{t h}}{\bar{\gamma}_{0}}}\right)-\frac{1-e^{-\frac{\sigma_{i}}{\rho_{i}} \gamma_{t h}}}{\sigma_{i} / \rho_{i}}+\sum_{k=1}^{L-1} \sum_{n=1}^{\left(\begin{array}{c}L-1 \\ k\end{array}\right)}\left(\frac{1-e^{-\gamma_{t h}\left(1 / \bar{\gamma}_{0}+\sum_{l=1}^{k} \varphi_{l, k, n}^{i}\right)}}{(-1)^{k}\left(\frac{1}{\bar{\gamma}_{0}}+\sum_{l=1}^{k} \varphi_{l, k, n}^{i}\right)}+\frac{1-e^{-\gamma_{t h}\left(\sigma_{i} / \rho_{i}+\sum_{l=1}^{k} \varphi_{l, k, n}^{i}\right)}}{(-1)^{k}\left(\frac{\sigma_{i}}{\rho_{i}}+\sum_{l=1}^{k} \varphi_{l, k, n}^{i}\right)}\right)\right]$

$$
\begin{aligned}
& \Omega_{i}\left(\gamma_{t h}\right)=\frac{4}{\rho_{i} \eta_{i}^{2}}\left[\bar{\gamma}_{0}\left(\ln \left(\frac{\eta_{i} \sqrt{\rho_{i}}}{2}\right)+u-1\right)\left(1-e^{-\gamma_{t h} / \bar{\gamma}_{0}}\right)+\frac{\rho_{i}}{\sigma_{i}}\left(1+\ln \left(\frac{2}{\sqrt{\rho_{i}}}\right)\right)\left(1-e^{-\gamma_{t h} \frac{\sigma_{i}}{\rho_{i}}}\right)+\eta_{i} \ln \left(\frac{2}{\sqrt{\rho_{i}}}\right) I_{2}\left(\frac{\sigma_{i}}{\rho_{i}}, \gamma_{t h}\right)\right. \\
& +I_{3}\left(\eta_{i}, 1 / \bar{\gamma}_{0}, \gamma_{t h}\right)+I_{4}\left(\eta_{i}, 1 / \bar{\gamma}_{0}, \gamma_{t h}\right)+\left(\ln \left(\frac{\eta_{i} \sqrt{\rho_{i}}}{2}\right)+u-1\right) \sum_{k=1}^{L-1}(-1)^{k} \sum_{n=1}^{\left({ }^{L-1} k\right.} \frac{1-\exp \left(-\gamma_{t h}\left(1 / \bar{\gamma}_{0}+\sum_{l=1}^{k} \varphi_{l, k, n}^{i}\right)\right)}{1 / \bar{\gamma}_{0}+\sum_{l=1}^{k} \varphi_{l, k, n}^{i}} \\
& +\left(1+\ln \left(\frac{2}{\sqrt{\rho_{i}}}\right)\right) \sum_{k=1}^{L-1} \sum_{n=1}^{(L-1)} \frac{1-\exp \left(-\gamma_{t h}\left(\frac{\sigma_{i}}{\rho_{i}}+\sum_{l=1}^{k} \varphi_{l, k, n}^{i}\right)\right)}{(-1)^{k}\left(\sigma_{i} / \rho_{i}+\sum_{l=1}^{k} \varphi_{l, k, n}^{i}\right)}+\eta_{i} \ln \left(\frac{2}{\sqrt{\rho_{i}}}\right) \sum_{k=1}^{L-1} \sum_{n=1}^{(L-1} \sum_{k}^{k} \frac{I_{2}\left(\frac{\sigma_{i}}{\rho_{i}}+\sum_{l=1}^{k} \varphi_{l, k, n}^{i}, \gamma_{t h}\right)}{(-1)^{k}} \\
& \left.+\sum_{k=1}^{L-1}(-1)^{k} \sum_{n=1}^{\left(\begin{array}{l}
L-1 \\
k
\end{array}\right)} I_{3}\left(\eta_{i}, \frac{1}{\bar{\gamma}_{0}}+\sum_{l=1}^{k} \varphi_{l, k, n}^{i}, \gamma_{t h}\right)+\sum_{k=1}^{L-1}(-1)^{k} \sum_{n=1}^{(L-1)} I_{4}\left(\eta_{i}, \frac{1}{\bar{\gamma}_{0}}+\sum_{l=1}^{k} \varphi_{l, k, n}^{i}, \gamma_{t h}\right)\right]
\end{aligned}
$$

where $f_{X}(\cdot)$ denotes the Gaussian PDF of $X$. In the high SNR region, combining (37) and (30) yields

$$
\begin{aligned}
P_{e} & =\frac{B}{\bar{\gamma}_{0} \sqrt{2 \pi}} \sum_{i=1}^{L} \int_{0}^{\infty}\left[\Xi_{i}\left(\frac{X^{2}}{\beta}\right)+\Omega_{i}\left(\frac{X^{2}}{\beta}\right)\right] e^{-\frac{X^{2}}{2}} d X \\
& =\frac{B}{2 \bar{\gamma}_{0}} \sqrt{\frac{\beta}{2 \pi}} \sum_{i=1}^{L} \int_{0}^{\infty}\left[\Xi_{i}(y)+\Omega_{i}(y)\right] y^{-\frac{1}{2}} e^{-\frac{\beta}{2} y} d y,
\end{aligned}
$$

where we have used the transformation $y=X^{2} / \beta$ to obtain the second equality. Substituting (31) and (32) into (38), a complicated expression for the ASEP is derived; in order to further simplify this expression, we utilize the following auxiliary functions

$$
\begin{gathered}
I_{5}(v, b)=\int_{0}^{\infty} y^{v-1} e^{-b y} d y=\frac{1}{b^{v}} \Gamma(v) \\
I_{6}(b, \varepsilon)=\int_{0}^{\infty} y^{-\frac{1}{2}} e^{-b y} \Gamma(0, \varepsilon y) d y \\
=\frac{2 \Gamma\left(\frac{1}{2}\right)}{\sqrt{b+\varepsilon}}{ }_{2} F_{1}\left(1, \frac{1}{2} ; \frac{3}{2} ; \frac{b}{b+\varepsilon}\right) \\
I_{7}(v, b)=\int_{0}^{\infty} y^{v-1} \ln (y) e^{-b y} d y=\frac{\Gamma(v)}{b^{v}}[\psi(v)-\ln (b)] \\
I_{8}(\varepsilon)=\int_{0}^{\infty} y^{-\frac{1}{2}} \ln (y) e^{-b y} \Gamma(2, \varepsilon y) d y \\
=I_{7}\left(\frac{1}{2}, b\right)+\varepsilon I_{7}\left(\frac{3}{2}, b+\varepsilon\right)
\end{gathered}
$$

where for the derivation of (39), (40), (41) and (42) we used [20, eq. (3.381.4)], [20, eq. (6.455.1)], [20, eq. (4.352.1)] and $[20$, eq. (8.352.2)] respectively. We note that due to space limitations, the closed-form expression for the ASEP in the high SNR region is omitted.

\section{NUMERICAL EXAMPLES}

In this section we compare the performance of the proposed relay-selection method with that of the BPS and the ESP scheme (mentioned in Sections III-C.1 and III-C.2, respectively), in terms of outage probability, ASEP and PHY-layer fairness attainment, when operating over independent Rayleigh fading channels. The results regarding the performance of the proposed system are also verified by a set of simulations, which as it is shown in Figs. 2-6 closely match the closed-form approximate results. In our examples, the average SNRs of the $S-R_{i}$ and $R_{i}-D$ links are considered to follow an exponential profile with decay factor denoted by $\delta$ and mean values equal to $\varepsilon_{1}$ and $\varepsilon_{2}$, respectively (i.e., $\bar{\gamma}_{S_{i}}=\alpha_{1} e^{-\delta(i-1)}, \quad \bar{\gamma}_{D_{i}}=\alpha_{2} e^{-\delta(i-1)}, i \in\{1, \ldots, L\}$, where $\alpha_{1}=L \varepsilon_{1}\left(e^{\delta}-1\right) /\left[\left(e^{\delta L}-1\right) e^{-\delta(L-1)}\right]$ and $\alpha_{2}=$ $L \varepsilon_{2}\left(e^{\delta}-1\right) /\left[\left(e^{\delta L}-1\right) e^{-\delta(L-1)}\right]$ are appropriately chosen constants, so that $\varepsilon_{1}$ and $\varepsilon_{2}$ do not depend on $L$ ). We note that this exponential decay on the average SNRs follows from the exponential path-loss model, implying linear spatial distribution of the participating relays. Furthermore, all relays are assumed to transmit with identical power $P_{S}$, whereas the average SNR $\bar{\gamma}_{0}$ of the direct $S-D$ channel is set equal to $10 \mathrm{~dB}$.

Fig. 2 depicts the outage probability of the above systems versus the number of available relays for $\gamma_{t h}=10 \mathrm{~dB}$, $\varepsilon_{1}=22 \mathrm{~dB}, \varepsilon_{2}=25 \mathrm{~dB}$ and $\delta=0.5$, while in Fig. 3 the same comparison in terms of ASEP (for BPSK modulation), is presented. The main result extracted from these figures is that the performance of the proposed scheme resembles that of the BPS system for small $L$, and that the difference in performance between these systems increases as $L$ grows large. Also, both the proposed and the BPS scheme outperform the ESP one.

An outage comparison of the above schemes when more disparate average SNRs are assumed $\left(\varepsilon_{1}=22 \mathrm{~dB}, \varepsilon_{2}=25 \mathrm{~dB}\right.$, $\delta=1$ ), is presented in Fig. 4. Apparently, the performance penalty of equally allocating the consumed power is evident for smaller $L$ in such scenario. Generally speaking, increasing 


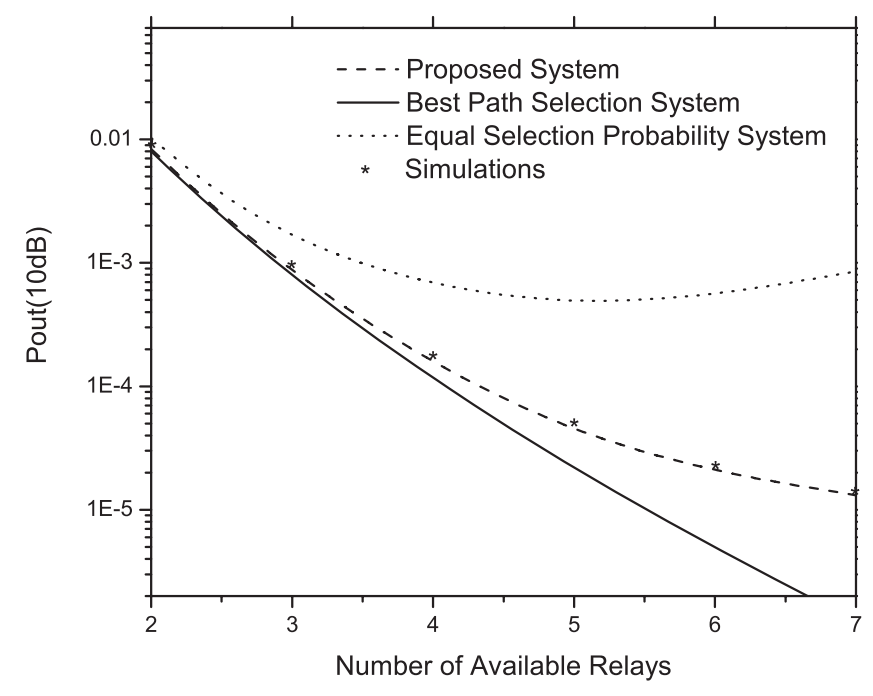

Fig. 2. Outage probability of the proposed, the BPS and the ESP scheme versus the number of available relays, for an SNR threshold equal to $10 \mathrm{~dB}$ and decay factor $\delta=0.5$.

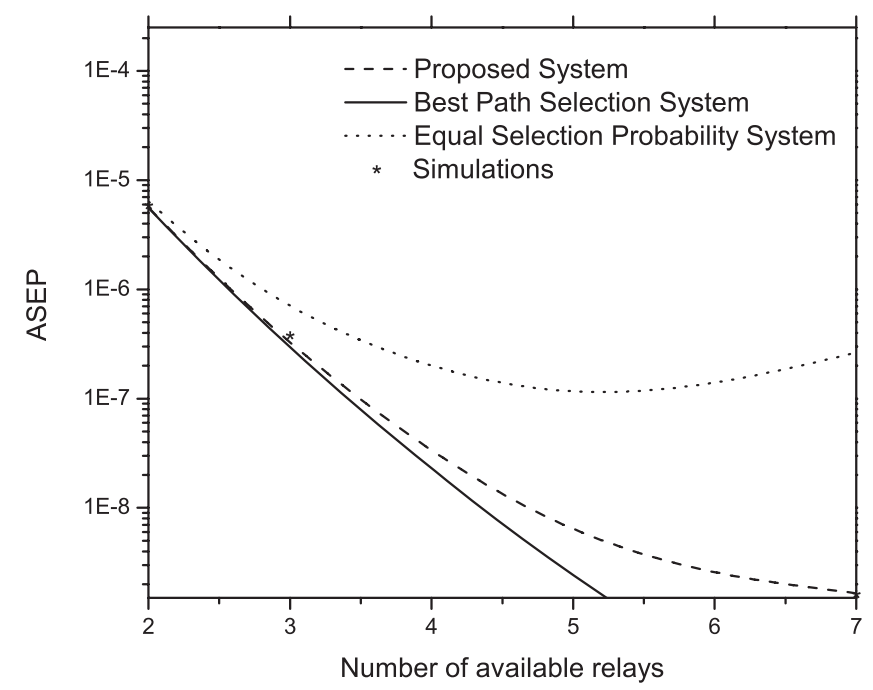

Fig. 3. ASEP for BPSK modulation of the proposed, the BPS and the ESP scheme, versus the number of available relays, assuming decay factor $\delta=0.5$.

the number of available relays leads to an increase in the diversity order of the BPS scheme, and both to an increase in the diversity order and to a performance degradation of the proposed and the ESP scheme; this degradation stems from the fact that increasing $L$ in an exponential-profile scenario implies that more relays with severe average fading conditions are also participating in the relaying process. Therefore, as we can see from Figs. 2-4, the cost of selecting the relay according to a PHY-layer fairness rule, instead of selecting the one with the best end-to-end SNR, is non-negligible only for relatively large (with respect to the disparateness of $\bar{\gamma}_{S_{i}}$ and $\bar{\gamma}_{D_{i}}$ ) number of available relays. Furthermore, the proposed scheme seems to outperform the ESP one, for each $L$ and for each realization of the disparateness of $\bar{\gamma}_{S_{i}}$ and $\bar{\gamma}_{D_{i}}$. Informally speaking, this fact can be explained considering that the relays with good average channel conditions are expected to consume less (see eq. (12)); consequently, the proposed selection method activates the relays with strong

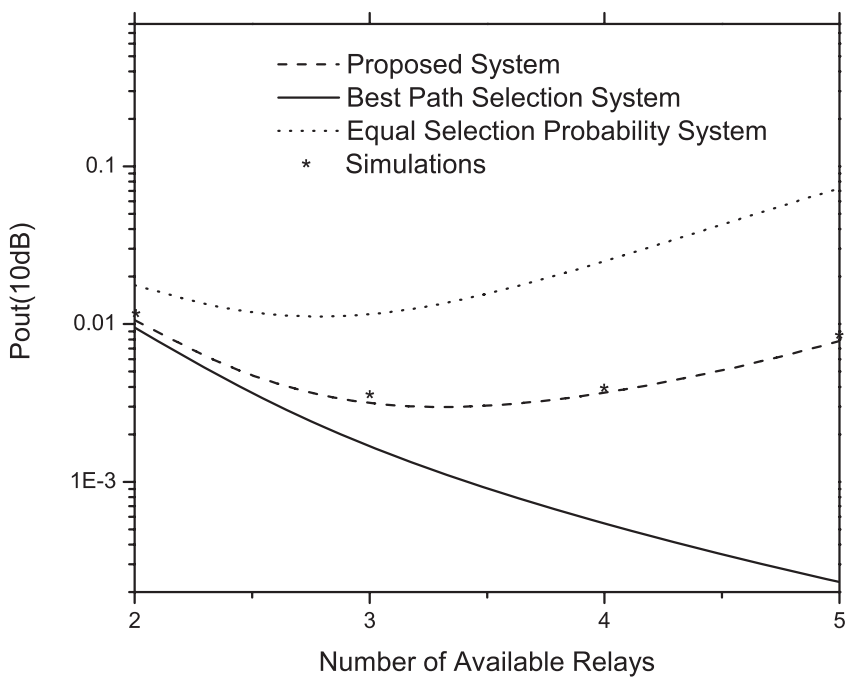

Fig. 4. Outage probability of the proposed, the BPS and the ESP scheme versus the number of available relays, for an SNR threshold equal to $10 \mathrm{~dB}$ and decay factor $\delta=1$.

channels for a greater fraction of time than those with weak channels, resulting thus in better performance compared to that of the ESP scheme.

In Fig. 5 the outage performance of the three compared schemes versus the normalized (with respect to $\gamma_{t h}$ ) $\varepsilon_{1}$ is depicted, for $L=3$ and $L=5$, assuming $\varepsilon_{2}=2 \varepsilon_{1}$ and $\delta=0.5$. As expected, the slope of the outage curves is the same for the three compared schemes, depending only on the number of available users, since the diversity order of these schemes is identical. Moreover, from the same figure it is evident that the proposed scheme tends to perform similarly as the BPS one in the high-SNR region, and that this resemblance in performance is very close even for low normalized average SNRs, when the number of available relays is small. Similar results regarding the relative performance of the three compared schemes are also derived from Fig. 6, where the ASEP performance for BPSK modulation versus $\varepsilon_{1}$, under the same channel assumptions (i.e., $\varepsilon_{2}=2 \varepsilon_{1}$ and $\delta=0.5$ ), is depicted. Notice that the ESP scheme appears again to perform worse than the proposed one, and that this difference in performance seems not to be significantly affected by the average channel conditions.

In Tables I and II, we provide a PHY-layer fairness attainment comparison of the three schemes mentioned above, for $\delta=0.5$ and $\delta=1$, respectively. The average SNR values $\varepsilon_{1}$ and $\varepsilon_{2}$ are set in each case equal to $22 \mathrm{~dB}$ and $25 \mathrm{~dB}$, respectively. We use the $\bar{P}_{\text {cons }_{i}}$ 's standard deviation (SD) over mean as a fairness metric, since it represents the most common dimensionless metric of the unevenness of the energy consumption distribution among the available relays. The mean value of the $\bar{P}_{\text {cons }_{i}}$ 's is also provided, as a percentage of $P_{S}$, for several values of $L$. We note that in Tables I and II the exact expression for the $\bar{P}_{\text {cons }_{i}}$ 's is used (eq. (13) together with (14)), and not the approximate one (eq. (18)); thus, the non-zero SD over mean for the proposed scheme reflects the impact of using (18) in (23), instead the exact expression for the $\bar{P}_{\text {cons }_{i}}$ 's. As we can see from Tables I and II, the proposed scheme's mean value of average power consumptions is the 


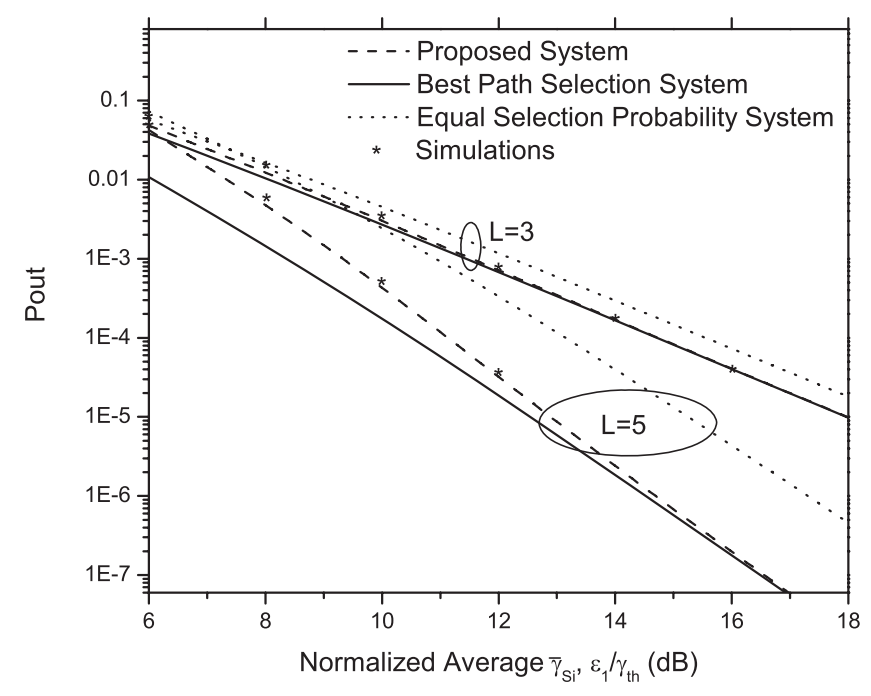

Fig. 5. Outage probability of the proposed, the BPS and the ESP scheme versus the average channel conditions.

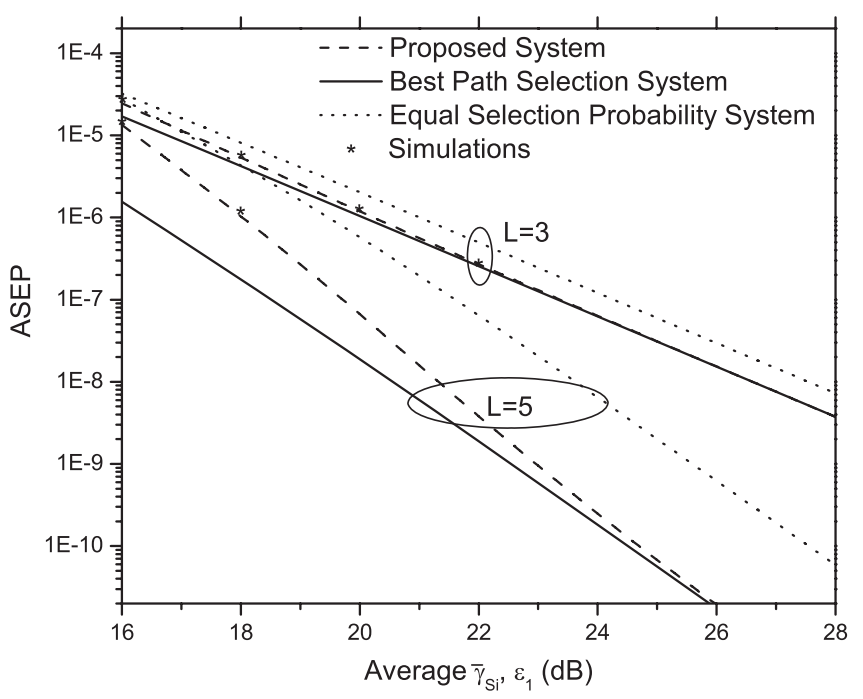

Fig. 6. ASEP for BPSK modulation of the proposed, the BPS and the ESP scheme, versus the average channel conditions.

nearly the same as that of the BPS scheme, and generally lower than that of the ESP. However, both the BPS and ESP schemes lead to a SD over mean of $\bar{P}_{\text {cons }_{i}}$ 's which is generally nonnegligible, and by far greater than the proposed scheme's. This verifies the assumption that the ESP and the BPS schemes are "non-fair" in terms of energy consumption, especially for large values of $P_{S}$. As expected, the SD over mean of $\bar{P}_{\text {cons }_{i}}$ 's increases for larger number of decay factor $\delta$, since the disparateness of $\bar{\gamma}_{S_{i}}$ and $\bar{\gamma}_{D_{i}}$ increases as well.

\section{CONCLUSION}

In this paper, we argued for the concept of fairness at the physical layer in amplify and forward cooperative diversity systems, followed by a selection method that utilizes knowledge on the average channel conditions in order to equitably allocate the total consumed energy among the available relaying terminals. Numerical results regarding the outage probability and ASEP showed that, in the high SNR region or when the number of available users is small, the above method performs similarly as the method where the terminal with the "best" end-to-end channel conditions is selected to relay for each coherence time interval.

\section{APPENDIX}

\section{DERIVATION OF (27) AND (35)}

The integral in (27) can be written as

$$
\begin{aligned}
I_{1}(\delta, b, \omega) & =\omega^{2} \int_{0}^{1} x \ln (\delta \omega x) e^{-b \omega x} d x \\
& =\omega^{2}\left[\ln (\delta \omega) \int_{0}^{1} x e^{-b \omega x} d x+\int_{0}^{1} x \ln (x) e^{-b \omega x} d x\right] \\
& =\omega^{2} \ln (\delta \omega)\left[\frac{e^{-b \omega}\left(-1-b \omega+e^{b \omega}\right)}{b^{2} \omega^{2}}\right. \\
& \left.+\int_{0}^{\infty} x \ln (x) e^{-b \omega x} d x-\int_{1}^{\infty} x \ln (x) e^{-b \omega x} d x\right] .
\end{aligned}
$$

Using [20, eq. (4.352.2)] and [20, eq. (4.358.1)], from (43) we infer

$$
\begin{aligned}
I_{1}(\delta, b, \omega)= & \frac{\ln (\delta \omega)}{b^{2}} e^{-b \omega}\left(-1-b \omega+e^{b \omega}\right) \\
& +\frac{1-\ln (b \omega)-u}{b^{2}}-\frac{e^{-b \omega}+\Gamma(0, b \omega)}{b^{2}},
\end{aligned}
$$

which in turn using the finite series representation for $\Gamma(2, \cdot)$ [20, eq. (8.352.2)] yields (27).

Likewise, using [20, eq. (8.352.2)], [20, eq. (4.331.1)], [20, eq. (4.331.2)] and [20, eq. (8.359.1)], the integral in (35) gives

$$
\begin{aligned}
& I_{4}(\varepsilon, b, \omega)=\int_{0}^{\omega} \ln (x) e^{-b x}(1+\varepsilon x) e^{-\varepsilon x} d x \\
& =\int_{0}^{1} \omega \ln (\omega x) e^{-(b+\varepsilon) \omega x} d x+\varepsilon I_{1}(1, b+\varepsilon, \omega) \\
& =-\frac{\Gamma(0,(b+\varepsilon) \omega)+\ln (b+\varepsilon)+\ln (\omega) e^{-(b+\varepsilon) \omega}+u}{b+\varepsilon} \\
& \quad+\varepsilon I_{1}(1, b+\varepsilon, \omega)
\end{aligned}
$$

Hence, (35) is derived by substituting (27) into (45).

\section{REFERENCES}

[1] J. N. Laneman, D. N. C. Tse, and G. W. Wornell, "Cooperative diversity in wireless networks: efficient protocols and outage behavior," IEEE Trans. Inform. Theory, vol. 50, pp. 3062-3080, Dec. 2004.

[2] J. N. Laneman, "Cooperative diversity in wireless networks: algorithms and architectures," Ph.D. dissertation, Massachusetts Institute of Technology, Cambridge, MA, Aug. 2002.

[3] J. N. Laneman and G. W. Wornell, "Distributed space-time-coded protocols for exploiting cooperative diversity in wireless networks," IEEE Trans. Inform. Theory, vol. 49, pp. 2415-2425, Nov. 2003.

[4] A. Høst-Madsen, "Capacity bounds for cooperative diversity," IEEE Trans. Inform. Theory, 2006.

[5] A. Høst-Madsen and J. Zhang, "Capacity bounds and power allocation for the wireless relay channel," IEEE Trans. Inform. Theory, 2005.

[6] A. Høst-Madsen, "Upper and lower bounds for channel capacity of asynchronous cooperative diversity networks," in Proc. 8th Annual Conference on Information Sciences and Systems, CISS 2004, Princeton, NJ, 2004.

[7] E. C. V. der Meulen, "Transmission of information in a T-terminal discrete memoryless channel," Ph.D. dissertation, University of California, Berkeley, CA, June 1968.

[8] T. Cover and A. E. Gamal, "Capacity theorems for the relay channel," IEEE Trans. Inform. Theory, vol. IT-25, pp. 572-584, Sept. 1979.

[9] A. Sendonaris, E. Erkip, and B. Aazhang, "User cooperation diversitypart I: system description," IEEE Trans. Commun., vol. 51, pp. 19271938, Nov. 2003. 
TABLE I

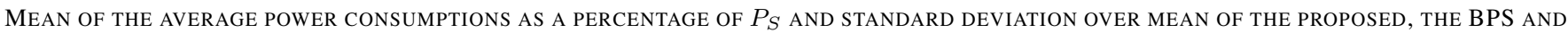
THE ESP SCHEME, FOR DECAY FACTOR $\delta=0.5$.

\begin{tabular}{|c|cc|cc|cc||}
\hline \hline \multicolumn{7}{||c||}{$\begin{array}{c}\text { Mean of the Average Power Consumptions as a percentage of } P_{S} \\
\text { and Standard Deviation over Mean }\end{array}$} \\
\hline \multirow{2}{*}{$L$} & \multicolumn{2}{|c|}{$\begin{array}{c}\text { Prop. } \\
\text { Scheme }\end{array}$} & \multicolumn{2}{c|}{ BPS } & \multicolumn{2}{l}{ ESP } \\
\hline \multirow{2}{*}{2} & Mean & SD/Mean & Mean & SD/Mean & Mean & SD/Mean \\
3 & $14.05 \%$ & $0.71 \%$ & $14.00 \%$ & $7.64 \%$ & $14.61 \%$ & $26.79 \%$ \\
5 & $6.65 \%$ & $3.12 \%$ & $6.55 \%$ & $17.71 \%$ & $7.88 \%$ & $53.38 \%$ \\
7 & $2.28 \%$ & $9.31 \%$ & $2.11 \%$ & $44.07 \%$ & $4.83 \%$ & $89.79 \%$ \\
\hline \hline
\end{tabular}

TABLE II

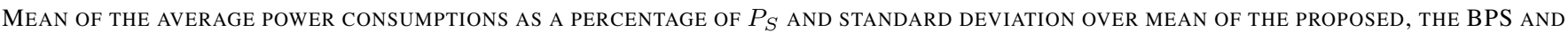
THE ESP SCHEME, FOR DECAY FACTOR $\delta=1$.

\begin{tabular}{|c|c|c|c|c|c|c|}
\hline \multicolumn{7}{|c|}{$\begin{array}{c}\text { Mean of the Average Power Consumptions as a percentage of } P_{S} \\
\text { and Standard Deviation over Mean }\end{array}$} \\
\hline \multirow[t]{2}{*}{$L$} & \multicolumn{2}{|c|}{$\begin{array}{c}\text { Prop. } \\
\text { Scheme }\end{array}$} & \multicolumn{2}{|c|}{ BPS } & \multicolumn{2}{|c|}{ ESP } \\
\hline & Mean & SD/Mean & Mean & SD/Mean & Mean & SD/Mean \\
\hline 2 & $14.56 \%$ & $1.55 \%$ & $14.34 \%$ & $16.70 \%$ & $16.67 \%$ & $47.84 \%$ \\
\hline 3 & $7.20 \%$ & $4.94 \%$ & $6.74 \%$ & $40.98 \%$ & $11.33 \%$ & $74.77 \%$ \\
\hline 5 & $2.64 \%$ & $11.87 \%$ & $2.11 \%$ & $83.01 \%$ & $8.68 \%$ & $83.85 \%$ \\
\hline 7 & $1.21 \%$ & $24.94 \%$ & $0.86 \%$ & $109.8 \%$ & $6.99 \%$ & $77.94 \%$ \\
\hline
\end{tabular}

[10] — - "User cooperation diversity-Part II: implementation aspects and performance analysis," IEEE Trans. Commun., vol. 51, pp. 1927-1938, Nov. 2003.

[11] K. Azarian, H. E. Gamal, and P. Schniter, "On the achievable diversitymultiplexing tradeoff in half-duplex cooperative channels," IEEE Trans. Inform. Theory, vol. 51, pp. 4152-4192, Dec. 2005.

[12] A. Bletsas, A. Khisti, D. P. Reed, and A. Lippman, "A simple cooperative diversity method based on network path selection," IEEE J. Selec. Areas Commun., vol. 24, pp. 659-672, Mar. 2006.

[13] G. Kramer, M. Gastpar, and P. Gupta, "Cooperative strategies and capacity theorems for relay networks," IEEE Trans. Inform. Theory, vol. 51, pp. 3037-3063, Sept. 2005.

[14] P. A. Anghel and M. Kaveh, "Exact symbol error probability of a cooperative network in a Rayleigh-fading environment," IEEE Trans. Wireless Commun., vol. 3, pp. 1416-1421, Sept. 2004.

[15] A. Ribeiro, X. Cai, and G. Giannakis, "Symbol error probabilities for general cooperative links," IEEE Trans. Wireless Commun., vol. 4, pp. 1264-1273, May 2005.

[16] P. A. Anghel, G. Leus, and M. Kaveh, "Multi-user space-time coding in cooperative networks," in Proc. International Conference on Acoustics, Speech, and Signal Processing (ICASSP 2003), Hong Kong, Apr. 2003, pp. 73-76.

[17] E. Beres and R. Adve, "On selection cooperation in distributed networks," in Proc. Conference on Information Sciences and Systems (CISS 06), Princeton, NJ, Mar. 2006.

[18] L. Dai, W. Chen, K. B. Letaief, and Z. Cao, "A fair multiuser cooperation protocol for increasing the throughput in energy-constrained adhoc networks," in Proc. International Conference on Communications (ICC'06), Istanbul, Turkey, June 2006.

[19] L. Dai and L. J. Cimini, "Improved fairness in energy-constrained cooperative ad-hoc networks," in Proc. Conference on Information Sciences and Systems (CISS 06), Princeton, NJ, Mar. 2006.
[20] I. S. Gradshteyn and I. M. Ryzhik, Table of Integrals, Series, and Products, 6th ed. New York: Academic, 2000.

[21] M. O. Hasna and M. S. Alouini, "End-to-end performance of transmission systems with relays over Rayleigh fading channels," IEEE Trans. Wireless Commun., vol. 2, pp. 1126-1131, Nov. 2003.

[22] R. You, H. Li, and Y. Bar-Ness, "Diversity combining with imperfect channel estimation," IEEE Trans. Commun., vol. 53, pp. 1655-1662, Oct. 2005 .

[23] J. H. Kotecha and A. M. Sayeed, "Transmit signal design for optimal estimation of correlated MIMO channels," IEEE Trans. Signal Processing, vol. 52, pp. 546-557, Feb. 2004

[24] M. Abramovitz and I. A. Stegun, Handbook of Mathematical Functions with Formulas, Graphs, and Mathematical Tables, 9th ed. New York: Dover, 1972.

[25] G. K. Karagiannidis, N. C. Sagias, and T. A. Tsiftsis, "Closed-form statistics for the sum of squared Nakagami- $m$ variates and its applications," IEEE Trans. Commun., vol. 54, pp. 1353-1359, Aug. 2006.

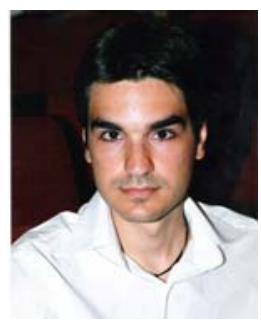

Diomidis S. Michalopoulos (S'05) was born in Thessaloniki, Greece, in 1983. He received the Diploma of Electrical and Computer Engineering from the Aristotle University of Thessaloniki in 2005, and since then he is pursuing a Ph.D degree in the ECE department. His research areas include digital communications over fading channels, with particular emphasis on the physical layer of Cooperative and Multihop Wireless Communications.

Mr. Michalopoulos is co-recipient of the Best Paper Award of the Wireless Communications Symposium (WCS) in 2007 IEEE International Conference on Communications (ICC'07). 


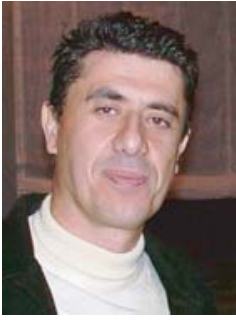

George K. Karagiannidis (M'97-SM'04) was born in Pithagorion Town, Samos Island, Greece. He received his university degree in 1987 and his $\mathrm{PhD}$ degree in 1999, both in Electrical Engineering, from the University of Patras, Patras, Greece.

From 2000 to 2004 he was Researcher at the Institute for Space Applications and Remote Sensing, National Observatory of Athens, Greece. In June 2004, he joined the faculty of Aristotle University of Thessaloniki, Greece where he is currently an Assistant Professor at the Electrical and Computer Engineering Department.

His major research interests include Wireless Communications Theory,
Digital Communications over Fading Channels, Cooperative Diversity Systems, Satellite Communications, Cognitive Radio and Free-Space Optical Communications.

Dr. Karagiannidis has published and presented more than 80 technical papers in scientific journals and international conferences, he is co-author in three chapters in books and also co-author in a Greek Edition Book on Mobile Communications. He is co-recipient of the Best Paper Award of the Wireless Communications Symposium (WCS) in 2007 IEEE International Conference on Communications (ICC'07). He is member of the Editorial Boards of IEEE Transactions on Communications, IEEE Communications Letters and EURASIP Journal on Wireless Communications and Networking. 